\title{
APPLICATION OF THE THEORY OF CONTINUOUS GROUPS TO A CERTAIN DIFFERENTIAL EQUATION.
}

BY MR. J. E. WRIGHT.

(Read before the American Mathematical Society, December 29, 1904.)

THE following question is propounded for solution in " Wolstenholme's Mathematical Problems" (No. 1964):

"Along the normal to a curve at $P$ is measured a fixed length $P Q ; O$ is a fixed point, and the curve is such that the circle described about $O P Q$ has a fixed tangent at $O$. Find the differential equation, the general integral, and the singular solution."

The main interest of the question lies in the fact that, although it was proposed without any reference to the theory of groups, it can be completely solved by straightforward application of that theory, and in addition it furnishes a good illustration of the points of connection between differential equations of the first order and that theory.

Take $O$ for origin, and the fixed tangent at $O$ for axis of $x$. Since $P Q$ is constant in length, it is clear that all lineal elements $a b$ satisfying the differential equation in question, and having their points on the circle $O P Q$, meet that circle at a constant angle.

Hence, if there exists a group whose path curves are $\left(x^{2}+y^{2}\right) / y=$ constant and which preserves angles, the differential equation admits that group.

Let $\xi \partial f / \partial x+\eta \partial f / \partial y$ be the symbol of the infinitesimal transformation of the group. We must have $\xi+i \eta=\phi(x+i y)$, since the transformation is conformal, and the differential equation of the path curves is $\xi d y-\eta d x=0$. is

The differential equation of the curves $\left(x^{2}+y^{2}\right) / y=$ constant

$$
2 x y d x-\left(x^{2}-y^{2}\right) d y=0 .
$$

Hence we must have $\xi /\left(x^{2}-y^{2}\right)=\eta / 2 x y$, and these are both equal to $(\xi+i \eta) /(x+i y)^{2}$. Therefore we take $\xi=x^{2}-y^{2}$ and $\eta=2 x y$, and all the conditions are satisfied. 
The finite equations of the group are given by integrating

$$
\frac{d x}{x^{2}-y^{2}}=\frac{d y}{2 x y}=d t \text {. }
$$

Of these equations we know the integral of the first, and therefore the remaining integral is given by a quadrature. This second integral is $x /\left(x^{2}+y^{2}\right)+t=$ constant.

Pursuing the general method, we take $\left(x^{2}+y^{2}\right) / y, x /\left(x^{2}+y^{2}\right)$, as new variables $Y, X$, respectively, and we know that the equation will reduce to the form $F\left(Y, Y^{\prime}\right)=0$.

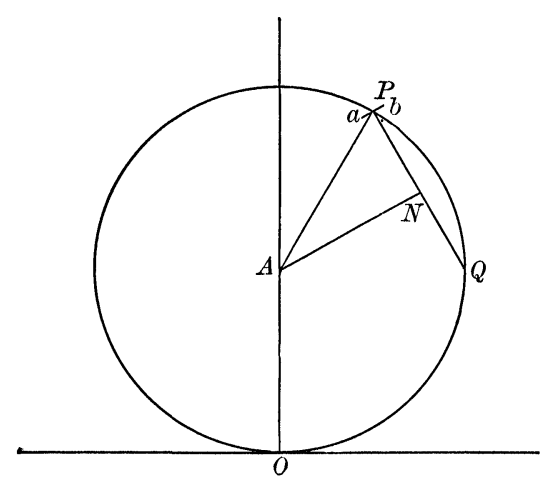

Let $P Q=c$, and let $r$ be the radius of the circle $O P Q$. Also let $p$ denote $d y / d x$. $A N$ is obviously parallel to $a b$, and therefore

$$
\tan ^{-1} \frac{y-r}{x}=\tan ^{-1} p+\tan ^{-1} \frac{c}{\sqrt{4 r^{2}-c^{2}}} .
$$

This gives as the differential equation

$$
\frac{c}{\sqrt{\left(\frac{x^{2}+y^{2}}{y}\right)^{2}-c^{2}}}=\frac{\left(y^{2}-x^{2}\right)-2 p x y}{p\left(y^{2}-x^{2}\right)+2 x y},
$$

which reduces to the form

Now

$$
x^{2}+2 x y p-y^{2}=c y \sqrt{1+p^{2}} .
$$

$$
\frac{d Y}{d x}=\frac{2 x y+p\left(y^{2}-x^{2}\right)}{y^{2}}, \quad \frac{d X}{d x}=\frac{\left(y^{2}-x^{2}\right)-2 p x y}{\left(x^{2}+y^{2}\right)^{2}} .
$$


Hence our differential equation becomes

$$
\frac{c}{\sqrt{Y^{2}-c^{2}}}=\frac{Y^{2}}{Y^{\prime}},
$$

the integral of which is

$$
\frac{\sqrt{Y^{2}-c^{2}}}{Y c}=X+\text { constant. }
$$

If we substitute the old variables, this becomes

$$
\left(x^{2}+y^{2}\right)\left(k^{2} c^{2}-1\right)+2 c^{2} k x+c^{2}=0,
$$

where $k$ is an arbitrary constant, and this is the required general integral.

Again, we know that if $f(x, y, p)=0$ admits the group $\xi f_{x}+\eta f_{y}, f(x, y, \eta / \xi)=0$ satisfies the equation, and contains as factor any singular solution of the equation. If there are any other factors, they are particular cases of the general integral for certain values of the arbitrary constant.

In our case $f(x, y, \eta / \xi)=0$ becomes

$$
\left[\left(x^{2}+y^{2}\right)^{2}-c^{2} y^{2}\right]\left(x^{2}+y^{2}\right)=0 .
$$

Here $x^{2}+y^{2}=0$ is the general integral when $k=\infty$, and

$$
\left(x^{2}+y^{2}\right)^{2}-c^{2} y^{2}=0
$$

is the singular solution.

BRYN MAWr, PA., October, 1904.

\title{
ON THE QUINTIC SCROLL HAVING A TACNODAL OR OSCNODAL CONIC.
}

\author{
BY PROFESSOR VIRGIL SNYDER.
}

(Read before the American Mathematical Society, October 29, 1904.)

Besides the quintic scrolls having three double conics which were discussed in the BuLLETIN (volume 9, pages 236-242), other particular types exist. Two of the double conics may become consecutive, forming a tacnodal conic ; or all three may become consecutive, forming an oscnodal conic. The necessary 